\title{
Ultra Low Dose CT Attenuation Correction Maps for Emission Computed Tomography
}

\author{
Haval Kadhem, Daniel Rodriguez, Jose-Rafael Tena, Kevin Wells, Emma Lewis and Matthew Guy
}

\begin{abstract}
We present a method for obtaining attenuation maps for use in emission computed tomography (ECT) using ultra low dose CT data (at $140 \mathrm{kVp}$, down to $10 \mathrm{~mA}$ ). This is achieved using a recursive k-means clustering method, the output of which initializes successive parameter-less region growing procedures. The method automatically produces templates corresponding to bone, lung, soft and dense tissue (muscle and fat). The segmentation of each tissue class from k-means clustering is used to compensate for the higher statistical noise variation seen at lower dose. The use of the region grower provides local contextual information that minimizes the impact of global noise. The templates were assigned appropriate linear attenuation coefficients and then convolved with the PET/SPECT system's PSF. This approach was applied to a dataset from an experimental anthropomorphic phantom exposed to systematically reducing CT dose derived from an X-ray beam at $140 \mathrm{kVp}$ and varying current from $160 \mathrm{~mA}$ (full diagnostic dose) to $10 \mathrm{~mA}$ (ultra low dose). Preliminary results show that for the purpose of CT attenuation correction, it is possible to successfully produce attenuation maps at ultra low dose with very low error (compared to full diagnostic dose) if used with the segmentation method presented.
\end{abstract}

\section{INTRODUCTION}

$\mathrm{E}_{\mathrm{n}}$ mission computed tomography (PET \& SPECT) represents non-invasive diagnostic imaging modalities used to assess the physiological or functional characteristics of suspected regions of pathology. In order to achieve quantitative accuracy, a number of corrections are often applied to the raw image data, including attenuation and scatter correction techniques.

Historically there are two popular approaches used to correct for attenuation: one is based on a direct measured correction and the other is based on a calculated correction. The calculated attenuation approach assumes a known outer body outline with constant attenuation properties [1], whereas the measured approach requires an additional scan, generally a transmission scan involving another radioactive source at higher photon energy, e.g. $662 \mathrm{keV}$.

With the advent of multi-modality PET/CT and SPECT/CT scanners, the use of CT data in emission attenuation correction is rapidly gaining use, due to the lower statistical noise, excellent image resolution and the dramatically reduced

This work has been supported by the Engineering and Physical Sciences Research Council (EPSRC), UK.

Haval Kadhem, Daniel Rodriguez, Jose-Rafael Tena, Kevin Wells and Emma Lewis, are with the School of Electronics \& Physical Sciences, University of Surrey, Guildford, Surrey GU2 5XH, UK.

(correspondence email: H.Kadhem@surrey.ac.uk)

Dr Matt Guy is with Nuclear Medicine, Royal Surrey County Hospital, Egerton Road, Guildford, Surrey GU2 7XX, UK. transmission acquisition times [2]. However, due to the relatively high additional radiation dosage from the CT scan, its use in attenuation correction is limited in situations where diagnostic dose may be important e.g. in pediatric imaging.

Since the observed intensities in CT images are reconstructed in Hounsfield Units (HU), they cannot be used directly for photon attenuation correction of emission data. Therefore precise conversion from CT value to linear attenuation coefficients at $511 \mathrm{keV}$ for PET (or $140 \mathrm{keV}$ for SPECT) is essential for accurate CT based attenuation correction. There are a number of methods for converting the reconstructed $\mathrm{CT}$ data for use as photon attenuation correction in emission data. The more common ones are segmentation based [3]-[6] and scaling based method [7]-[10].

Segmentation based methods divide the CT image into different tissue types and replaces the CT values of each type with the appropriate attenuation coefficient at $511 \mathrm{keV}$. The Bilinear [9] and Hybrid [2] scaling methods use scaling factors to transforms the $\mathrm{CT}$ values in $\mathrm{HU}$ to linear attenuation coefficients at the energy of the PET scanner via a bi-linear function defined by (1)

$\mu_{x}(E)= \begin{cases}f_{1}(x)=0.0961 \times x & \text { for } x<0 H U \\ f_{2}(x)=0.0961+5.08 \times 10^{-5} \times x & \text { for } x>0 H U\end{cases}$

where $f_{1}(x)$ represents mixing between soft tissue and lungs, while $f_{2}(x)$ represents mixing of soft tissue and bone. However, the Bilinear/Hybrid Scaling methods do not perform well for CT enhanced contrast agents and if non biological materials are present [2].

In this paper we present a method for obtaining an ultra low dose CT attenuation correction (AC) map using k-means clustering combined with an automatically-initialized regiongrowing based segmentation technique. This segmentation method produces binary templates corresponding to bones, lungs, soft tissue and dense tissue (muscle and fat), subsequently labeled using prior knowledge of the HU numbers within each template. The advantage of using this approach is that hard region based segmentation of the tissues can be used as a basis for compensation of the higher statistical noise variation seen at the lower dose. 


\section{MAterials AND METHOD}

\section{A. Data acquisition.}

A tissue-equivalent anthropomorphic phantom, containing materials with attenuation characteristics corresponding to cortical and spongiosa bones, lungs, soft and dense tissue was prepared for CT scanning at various X-ray beam currents.

CT scans of the anthropomorphic phantom were acquired with a GE Medical Systems (GEMS) Discovery LS PET/CT scanner, using a standard clinical 'soft' filter and imaging parameters: $1.5: 1$ pitch, $512 \times 512$ image grid on a $1 \times 1 \times 5 \mathrm{~mm}$ grid size and $140 \mathrm{kVp}$, with varying beam current. In order to assess the lowest possible beam current which produces acceptable attenuation map, nine $\mathrm{CT}$ scans were acquired at $160,80,70,60,50,40,30,20$ and $10 \mathrm{~mA} .10 \mathrm{~mA}$ was the lowest allowed $\mathrm{x}$-ray beam current setting for the scanner. The dose-length product (DLP) for the $160 \mathrm{~mA}$ scan was 513.80 mGy.cm and $32.11 \mathrm{mGy} . \mathrm{cm}$ for the $10 \mathrm{~mA}$ scan.

\section{B. Target Object Isolation.}

For each dataset an exemplar slice containing a wide range of tissue types, was chosen as the start image $I_{0}$. A k-means clustering method [11] was initially used to isolate the target object (phantom) from the scanner's gantry and bed. This was achieved by applying a three class k-means segmentation and multiplying the resulting binary template of the phantom with $I_{0}$. The resulting image is referred to as $I_{1}$; an exemplar slice from the $10 \mathrm{~mA}$ dataset and the result of the target isolation is shown in fig 1 .
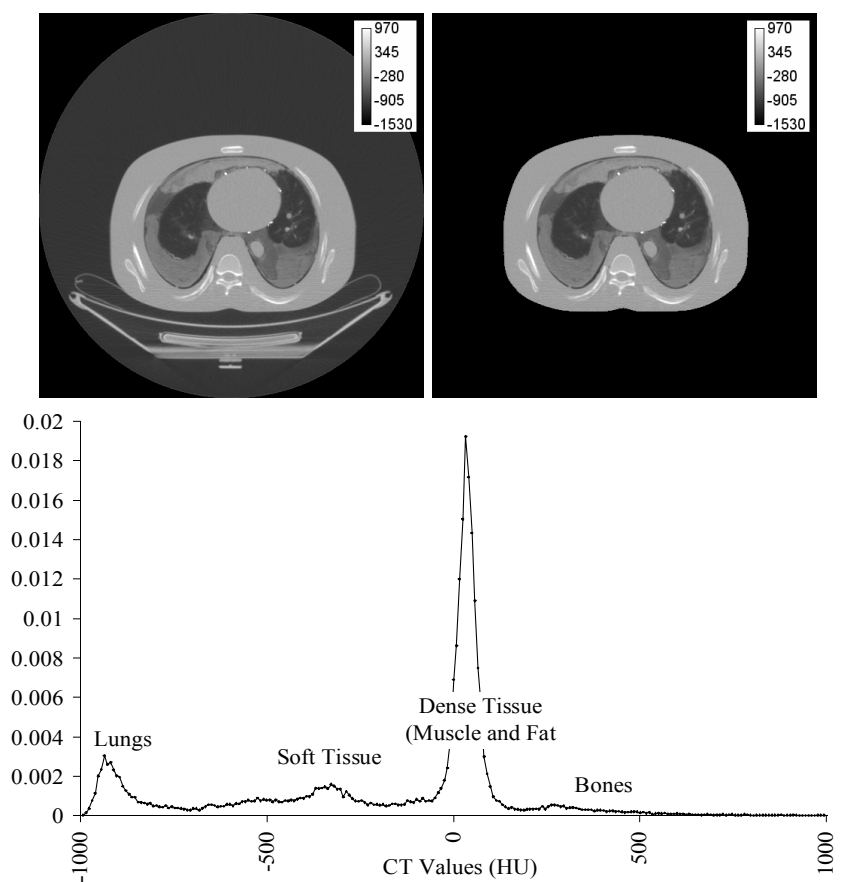

Fig. 1. Top Left: Exemplar slice through CT Phantom data. Top Right: The phantom is isolated and the scanner bed and gantry removed and assigned the values of the background. Bottom: image histogram of the isolated phantom data, corresponding to the right top hand image.

\section{Tissue Template Generation.}

The isolated image, $I_{1}$ was then segmented into 5 different regions again using the k-means technique, but now set to a 5 cluster segmentation. The resulting templates thus correspond to the expected intensity ranges from known Hounsfield Units of bones, lungs, soft tissue, dense tissue and the background. The image intensity values in $I_{1}$ are grouped by their proximity to the centroids and the results are stored as an image $I_{2}$, containing a unique label for each tissue type. Mathematical erosion is then applied to each template. This ensures over spill is subsequently avoided during region growing, particularly where partial volumes occur at the edges of different tissue classes. The resulting templates were then used to automatically initialize seed regions on $I_{1}$ for a parameter-less region growing tool [12],[13]. A screen shot of the region grower interface is shown in fig 2 .

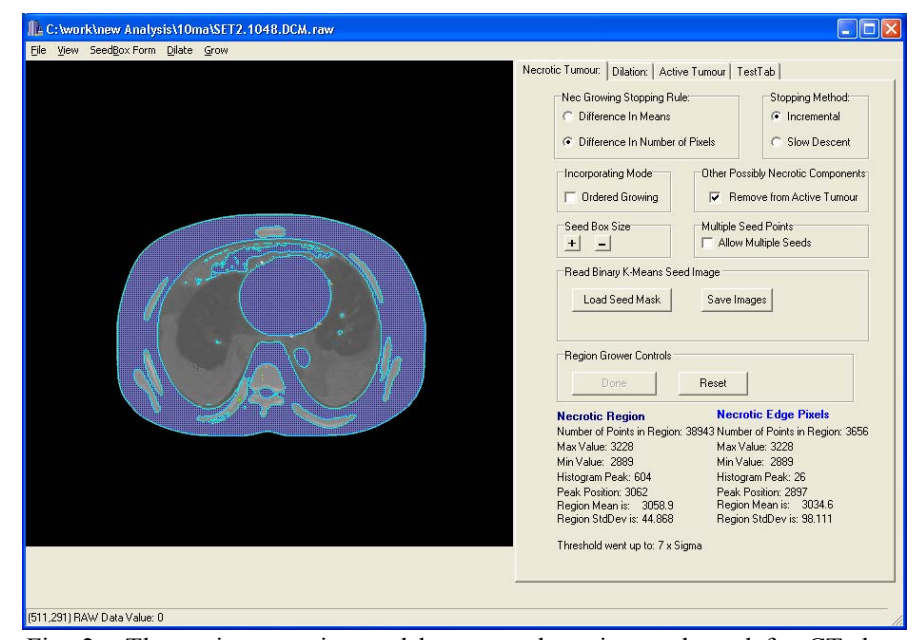

Fig. 2. The region growing and k-means clustering tool used for CT data segmentation into different templates. The eroded dense tissue template was used here as a seed region and the final dense tissue template region is shown.

The region grower tool computes the mean and variance of the initial seed region, and then iteratively appends voxels using a 2-D 9-way connectivity constraint (26-way in 3D) which satisfies an automatically defined intensity window initialized in (2).

$$
W=\mu \pm 0.5 \sigma
$$

where $W$ is the intensity window, $\mu$ is the seed region mean and $\sigma$ is the standard deviation of the seed region. The width of the intensity window is systematically incremented at each iteration step by changing the lower or higher intensity threshold.

The region growing process is halted when the algorithm detects a sudden change in the mean or variance, corresponding to the point at which the grown region has flooded outside the target region and into an adjoining region. The result of the previous iteration, prior to this fill change, is then stored. The region grower also has the ability to iterate around this critical point, and thus overcomes gross region underestimates caused by potentially large incremental step 
size. The resulting grown regions were stored as templates for the five different tissue types of interest, $I_{3}$ for Lung, $I_{4}$ for Bone, $I_{5}$ for soft tissue and $I_{6}$ for dense tissue.

The purpose of the region grower is to provide a refinement on the tissue templates produced by globally thresholding the intensity values via the k-means algorithm. The region grower thus utilizes local information (i.e. at the grown region boundary) to determine a close to optimal threshold that can be used, and in this way minimizes the effects of noise on the segmentation process in lower dose data.

Given that the bone regions were not spatially connected, the region grower was used with the option of including regions containing similar statistical values. The soft tissue template could have been segmented further by using a larger number of clusters; however that would produce negligible difference in attenuation at $511 \mathrm{keV}$. The bone and lungs templates were then segmented into 2 and 3 further segmentations respectively by using a further round of kmeans clustering. This further segmentation is needed to obtain a more accurate representation of tissue attenuation at these regions of continuously varying densities corresponding to cortical/spongiosa bone for the bone template and major pulmonary vessels, plus two further templates of varying density corresponding to airways and functional tissue in the lungs template.

\section{Attenuation map generation.}

CT-based attenuation maps were generated at $511 \mathrm{keV}$ for PET by assigning known linear attenuation coefficients (LAC) at $511 \mathrm{keV}$, for the different tissue templates. The values used for the LAC, were read from the bilinear function used by the Discovery scanner and from [14]. A composite image $I_{7}$ was generated and convolved with a 4-mm full width half maximum (FWHM) Gaussian filter [15], simulating the point spread function (PSF) of the GE Advance Nxi PET system. The resulting processed image was then spatially down sampled in order to match the acquired image grid of the PET scanner. The final image was then stored as the attenuation map, $I_{8}$. A block diagram of the steps involved in generating the templates for the attenuation map can be seen in fig 3.

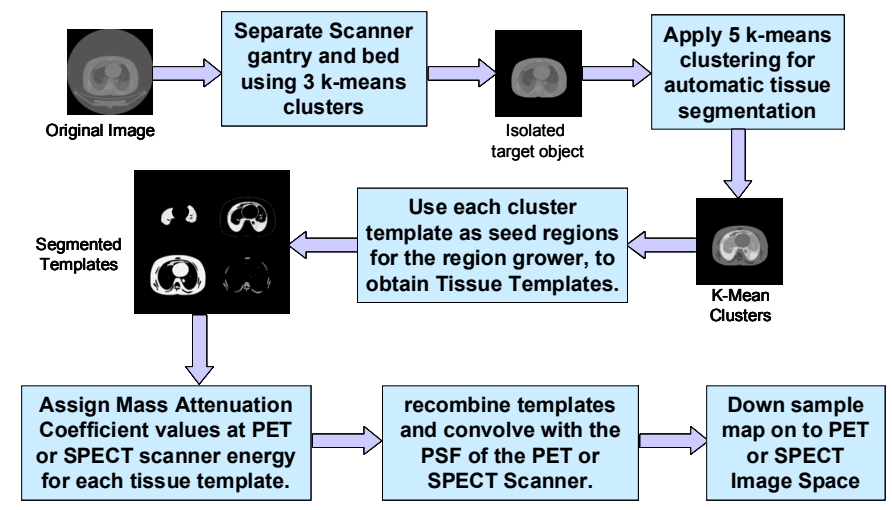

Fig. 3. Overview of the steps to produce attenuation maps via the segmentation method by k-means and region growing.

\section{E. Data analysis.}

To evaluate the effect of lowering of the CT x-ray beam current on the noise property of the data, the main templates generated by the k-means clustering were used as Regions of Interests (ROIs) and the statistical properties of the data were calculated. The mean $\mathrm{CT}$ value in the region was divided by the standard deviation of each template to obtain the Signal to Noise ratio (SNR). The final attenuation maps generated for the various doses were compared to the ground truth attenuation map generated from the standard clinical current of $160 \mathrm{~mA}$ using the sum of squared difference (SSD) measure.

\section{Preliminary Results \& Analysis}

Fig 4 shows the tissue templates generated for slice 48 for the $10 \mathrm{~mA}$ data.
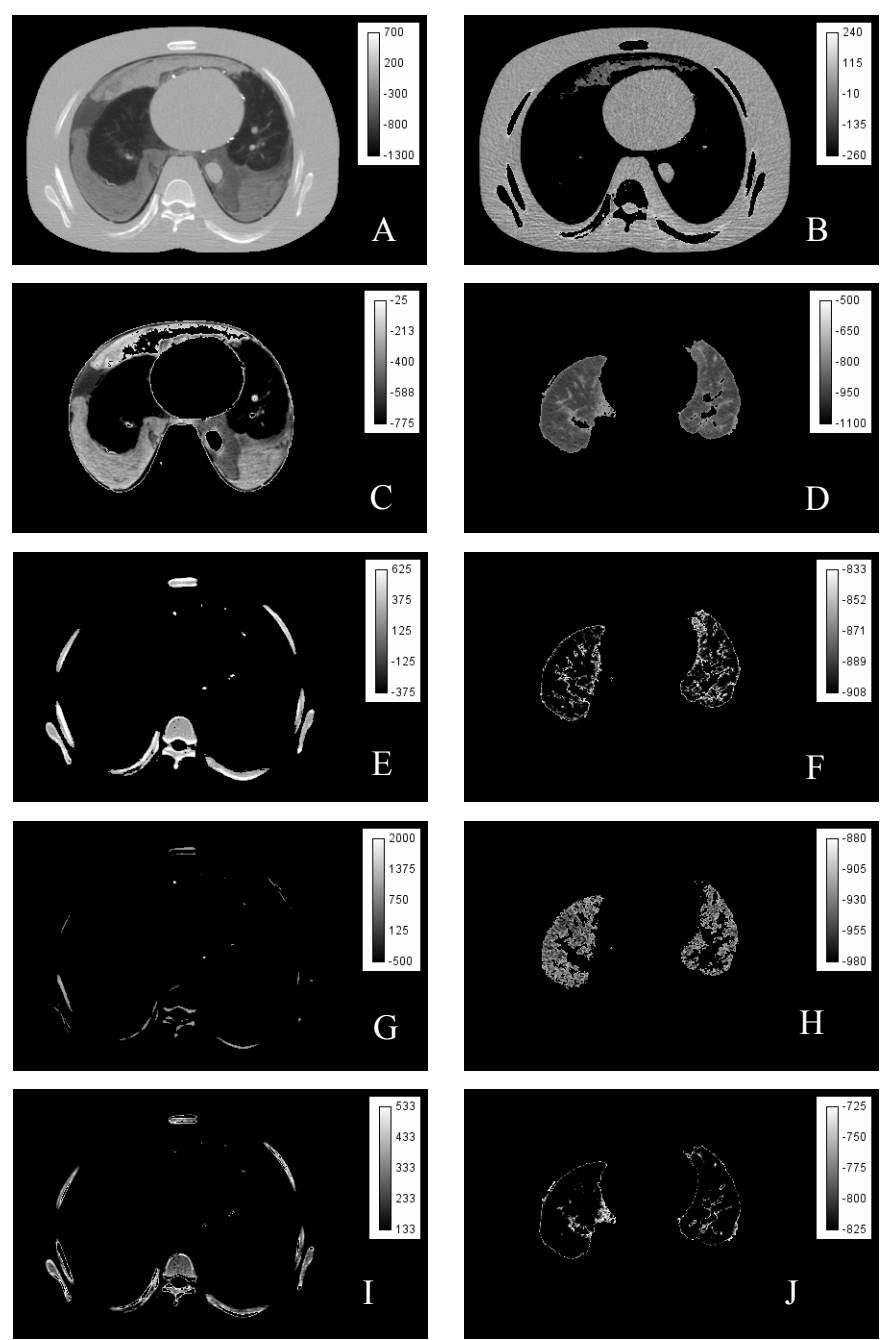

Fig. 4. Result of the k-means initialized region grower, automatically generated templates overlaid on original $10 \mathrm{~mA}$ exemplar slice data. (a) $10 \mathrm{~mA}$ CT image, (b) dense tissue, (c) soft tissue, (d) lungs, (e) bones, (f) large airways of lung, (g) cortical bone, (h) small airways of lung, (i) spongiosa bone and (j) main pulmonary vessels in the lungs.

The dense tissue template was observed to be most affected by beam hardening, streak artifacts from the reconstruction algorithm and scatter induced artifacts at lower x-ray beam 
currents as shown in fig 5. However, since each tissue template generated will have a single linear attenuation coefficient related to the $\mathrm{HU}$ of the mean value for that template, these artifacts do not propagate to the attenuation correction map.

This is a potential performance advantage over the common scaling methods such as the bilinear and the hybrid method particularly at low dose CT scans where the standard deviation of the CT values influences the attenuation values.

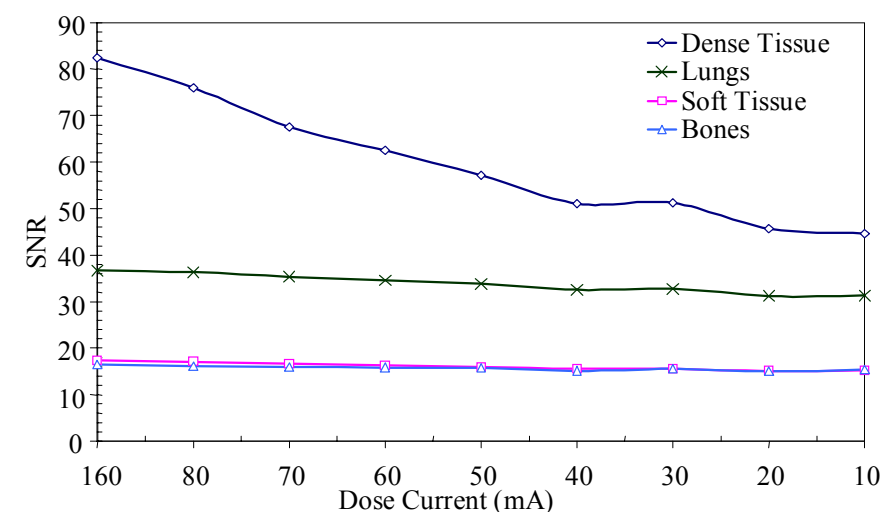

Fig. 5. Signal to noise ratio (SNR) variation with dose current for the different tissue templates. The Dense Tissue template is greatly affected by the lowering of the dose due to an observed increase in the streak artifacts of the reconstruction algorithm. Note, $\mathrm{x}$-axis is not linearly scaled.

The results of some of the generated attenuation maps are presented in fig 6 . The quality of the lower current CT scans appears noisier compared to that of the standard clinical scan at $160 \mathrm{~mA}$. However the down sampling onto the PET image grid and PSF filtering will produce very similar attenuation maps and thus reduce variations due to noise.

The final attenuation maps generated were compared with that of the attenuation map generated using the standard clinical CT data acquired at $160 \mathrm{~mA}$; the results of the sum of squared difference measure is shown in Table 1.

TABLE 1

SUM OF SQUARES DIFFERENCE BETWEEN THE ATTENUATION MAP AT HIGH DOSE AND THE LOWER DOSE ATTENUATION MAPS.

\begin{tabular}{cccc} 
AC Map at & $\begin{array}{c}\text { Segmented \& } \\
\text { labeled }\end{array}$ & $\begin{array}{c}\text { Convolved with } \\
\text { PSF of PET }\end{array}$ & $\begin{array}{c}\text { Down sampled on } \\
\text { PET Image Space }\end{array}$ \\
\hline $10 \mathrm{~mA}$ & 93.75 & 69.88 & 24.75 \\
$20 \mathrm{~mA}$ & 98.82 & 76.88 & 27.19 \\
$30 \mathrm{~mA}$ & 77.03 & 58.29 & 20.75 \\
$40 \mathrm{~mA}$ & 114.75 & 94.49 & 34.02 \\
$50 \mathrm{~mA}$ & 53.65 & 42.46 & 15.10 \\
$60 \mathrm{~mA}$ & 41.35 & 32.84 & 11.66 \\
$70 \mathrm{~mA}$ & 29.60 & 23.24 & 8.28 \\
$80 \mathrm{~mA}$ & 14.30 & 11.47 & 4.09 \\
$160 \mathrm{~mA}$ & 0.00 & 0.00 & 0.00 \\
\hline
\end{tabular}

A number of factors affect the SSD error including registration errors and Partial Volume Effects (PVEs). Partial Volume Effects (PVEs) were observed around the bones and at the boundaries of the phantom. The PVEs appear more prominent at $\mathrm{CT}$ acquisitions taken at lower x-ray current and are in turn propagated to the AC Map. However the magnitude of this problem is reduced by down sampling of the CT image to the PET grid size of $128 \times 128$.

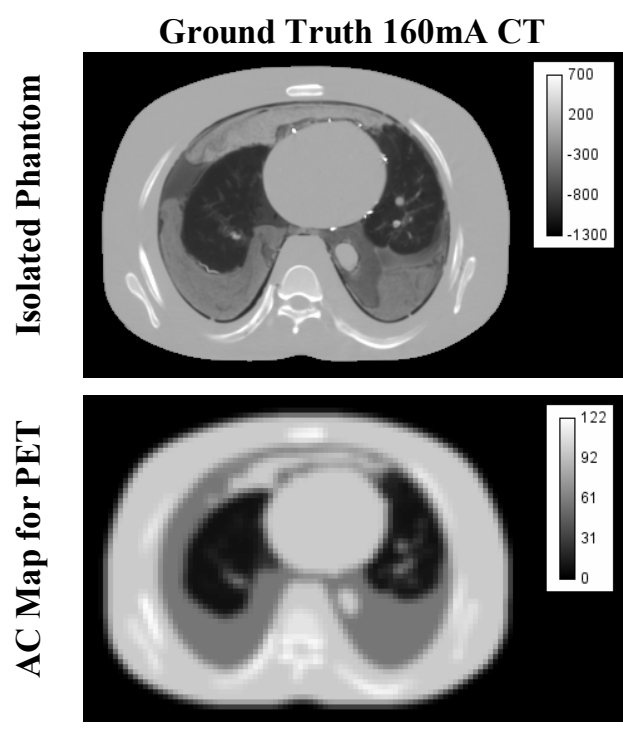

Fig. 6. Attenuation map results for PET. Top row: exemplar slice of the thorax acquired at $160 \mathrm{~mA}, 40 \mathrm{~mA}$ and $10 \mathrm{~mA} \mathrm{CT}$. Middle row: final attenuation map generated for PET data. Bottom row: the pixel intensity difference $(\%)$, between the $160 \mathrm{~mA}$ ground truth $\mathrm{AC}$ map and the corresponding AC maps generated at lower doses. Note, maximum difference is $<0.4 \%$ demonstrating excellent agreement with AC map produced using full clinical dose.
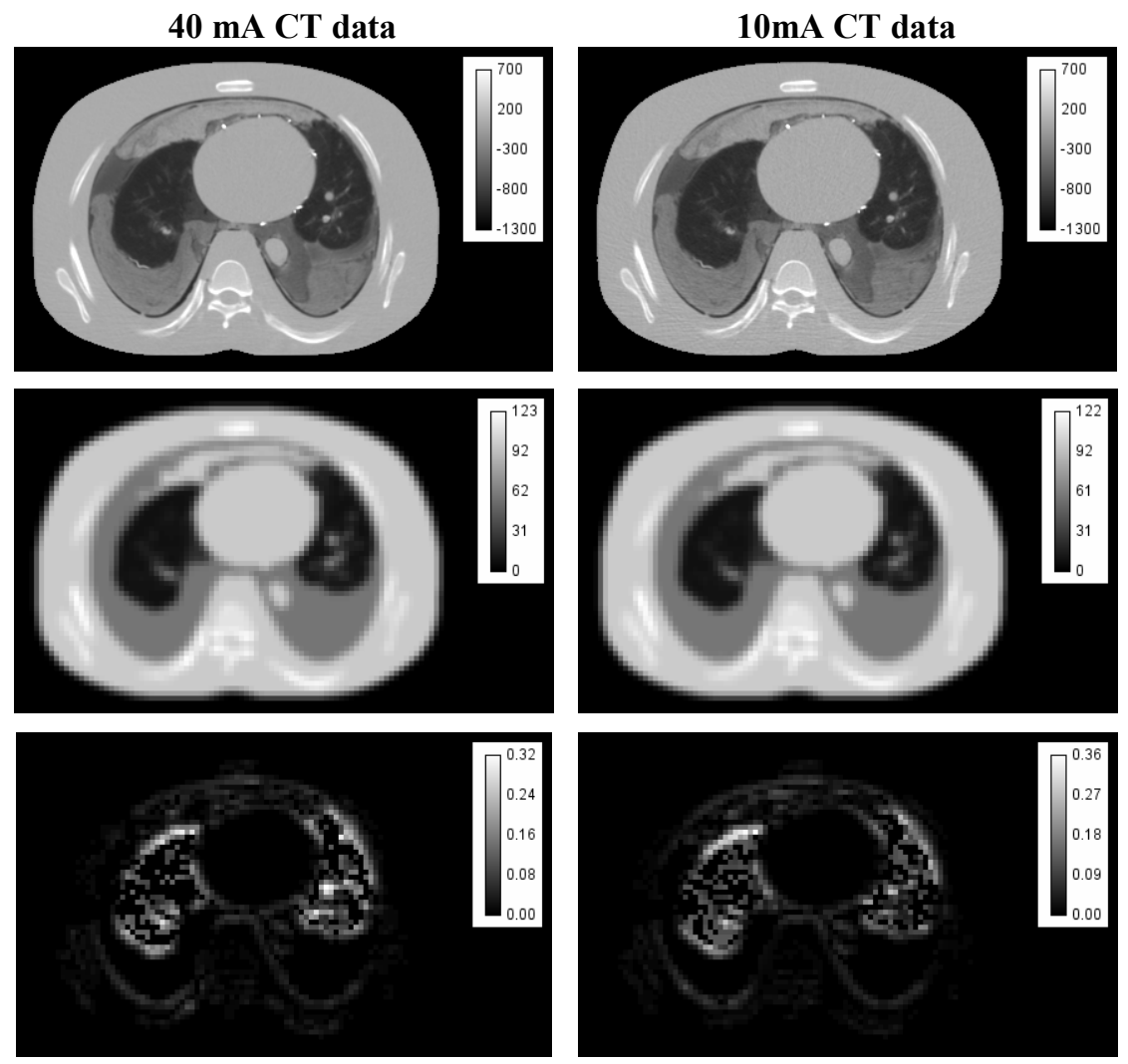


\section{CONCLUSIONS}

We demonstrated in a phantom study that ultra low dose CT scan at $140 \mathrm{kVp}$ and $10 \mathrm{~mA}$ x-ray beam settings can be used as a near perfect attenuation map for correction of emission data, compared to standard clinical dose.

Segmentation using k-means clustering and region growing can be used to generate suitable templates for Attenuation labeling and largely overcomes the increase in noise in the lower dose CT scans. Beam hardening and scatter induced artifacts do not appear to propagate to the AC maps in this method.

The advantage of combining k-means templates with parameter-less region growing are (i) attenuation maps can be generated automatically; (ii) the method appears robust and able to tolerate large amounts of noise and image artifact and (iii) thus provides attenuation maps at more than $\mathrm{x} 10$ reduction in dose.

The lowest relative dose (much less than $10 \mathrm{~mA}$ ) where the method can still produce acceptable attenuation maps needs to be determined. Further tests are planned to assess the technique on real patient data.

\section{ACKNOWLEDGMENT}

The assistance of Lodestone Diagnostic Imaging Ltd, Guildford, UK is gratefully acknowledged. H. Kadhem would also like to thank the Engineering and Physical Sciences Research Council, UK for partial funding of this work.

\section{REFERENCES}

[1] T. G. Turkington, "Introduction to PET Instrumentation", J Nucl Med Technol, vol. 29, pp. 4-11, 2001.

[2] P. E. Kinahan, D. W. Townsend, T. Beyer and D. Sashin, "Attenuation correction for a combined 3D PET/CT scanner", Medical Physics, vol. 25, pp 2046-53, 1998.

[3] S. R. Meikle, M. Dahlbom, and S. R. Cherry, "Attenuation correction using count-limited transmission data in positron emission tomography", J. Nucl. Med, vol. 34, pp 143-150, 1993.

[4] M. Xu, P. D. Cutler, and W. K. Luk, "Adaptive, segmented attenuation correction for whole-body PET". IEEE Trans Nucl Sci, vol. 43, pp. 331-336, 1996.

[5] V. Bettinardi, E. Pagani, M. C. Gilardi, C. Landoni, C. Riddell, G. Rizzo, and et al, "An automatic classification technique for attenuation correction in positron emission tomography". Eur J Nucl Med, vol. 26, pp. 447-458, 1999.

[6] H. Zaidi, M. Diaz-Gomez, A. E. Boudraa, and D. O. Slosman, "Fuzzy clustering-based segmented attenuation correction in wholebody PET imaging", Phys. Med. Biol, vol. 47, pp. 1143-1160, 2002.

[7] T. Beyer, P. E. Kinahan, D. W. Townsend, and D. Sashin, "The use of x-ray CT for attenuation correction of PET data", conference record of the IEEE Nuclear Science Symposium and Medical Imaging Conference, vol. 4, pp. 1573-1577, 1994.

[8] S. C. Blankespoor, X. Xu, K. Kaiki, J. K Brown, H. R. Tang, C.E. Cann, and et al., "Attenuation correction of SPECT using x-ray CT on an emission-transmission CT system: Myocardial perfusion assessment", IEEE Trans. Nucl. Sci, vol. 43, pp. 2263-2274, 1996.

[9] C. Burger, G. Goerres, S. Schoenes, A. Buck, A. H. R. Lonn, and G. K. Von Schulthess, "PET attenuation coefficients from CT images: Experimental evaluation of the transformation of CT into PET 511keV attenuation coefficients", Eur J Nucl Med Mol Imag, vol. 29, pp. 922-927, 2002
[10] K. J. LaCroix, B. M. W Tsui, B. H. Hasegawa, et al, "Investigation of the use of X-ray CT images for attenuation compensation in SPECT", IEEE Trans Nucl Sci, vol. 41, pp. 2793-2799, 1994.

[11] A. K. Jain, and R. C. Dubes, "Algorithms for Clustering Data", Prentice Hall, 1988.

[12] D. Rodriguez, "Tumour volume quantification in childhood cancer with application to Wilms' tumours", MRes Dissertation at Centre for Vision, Speech and Signal Processing (CVSSP), University of Surrey, Guildford, 2003.

[13] J. R. Tena, "Segmentation methods for Wilm's tumour CT data", MSc Dissertation at CVSSP, University of Surrey, Guildford, 2004.

[14] J. H. Hubbell, and S. M. Seltzer, "Tables of X-Ray Mass Attenuation Coefficients and Mass Energy-Absorption Coefficients", NIST, Gaithersburg, MD 20899, http://physics.nist.gov/PhysRefData/ XrayMassCoef/tab4.html.

[15] G. Tarantola, F. Zito, P. Gerundini, "PET Instrumentation and Reconstruction Algorithms in Whole-Body Applications", $J$ Nucl Med. vol. 44, pp. 756-769, 2003. 